other case visual disturbance and mental deterioration remained unimproved.

From the angiographic findings mentioned above, the abnormal vascular network is thought to be collateral circulatory pathways developing around the site of oclusion. In the beginning of complete occlusion, the development of the network was very poor, and the cerebral arteries were hardly visualized. Then, the network developed gradually and cerebral arteries became to be more clearly seen.

Transient ischemic attacks prior to the rnore persistent paresis might occur by means of partial occlusion of the artery. When the stenosis became to be complete, the neurological symptoms were irreversible. However, in the younger patient, extensive collateral circulation developed in the early stage. Therefore, neurological symptoms except a few signs, were unexpectedly reversible. The real cause of such vascular occlusion was still obscure, but angiitis due to unknown origin must be studied because of the fact that fever was detected at the onset of the more persistent symptoms and steroid hormone was clinically effective in one case.

\title{
D-12. On the Cases with Abnormal Vascular Network in the Cerebral Basal Region in the United States
}

\author{
J. Lawrence Pool, Ernst H. Wood and Yutaka MaKi \\ Neurological Institute in New York
}

A study of abnormal vascular network in the cerebral basal region was undertaken in the United States under our direction. A case, as an example of abnormal vascular network, was reported by one of the Authors at the meeting of the 6th Japanese Neurological Association in 1965. Copies of the angiograms, as well as a summary of an autopsied case, were sent to each of the 113 Neurosurgical program Directors in the U.S. Also submitted along with the above mentioned summaries were questionnaires in regard to the experiences of the various with similar vascular lesions.

Up to September $30,1966,62$ replies to the questionnaires were received. The results were as follows:

1) Their opinions about our cases were divided as follows:

25 due to congenital origin

19 due to non-congenital

2 either congenital or non-congenital origin

16 unknown 


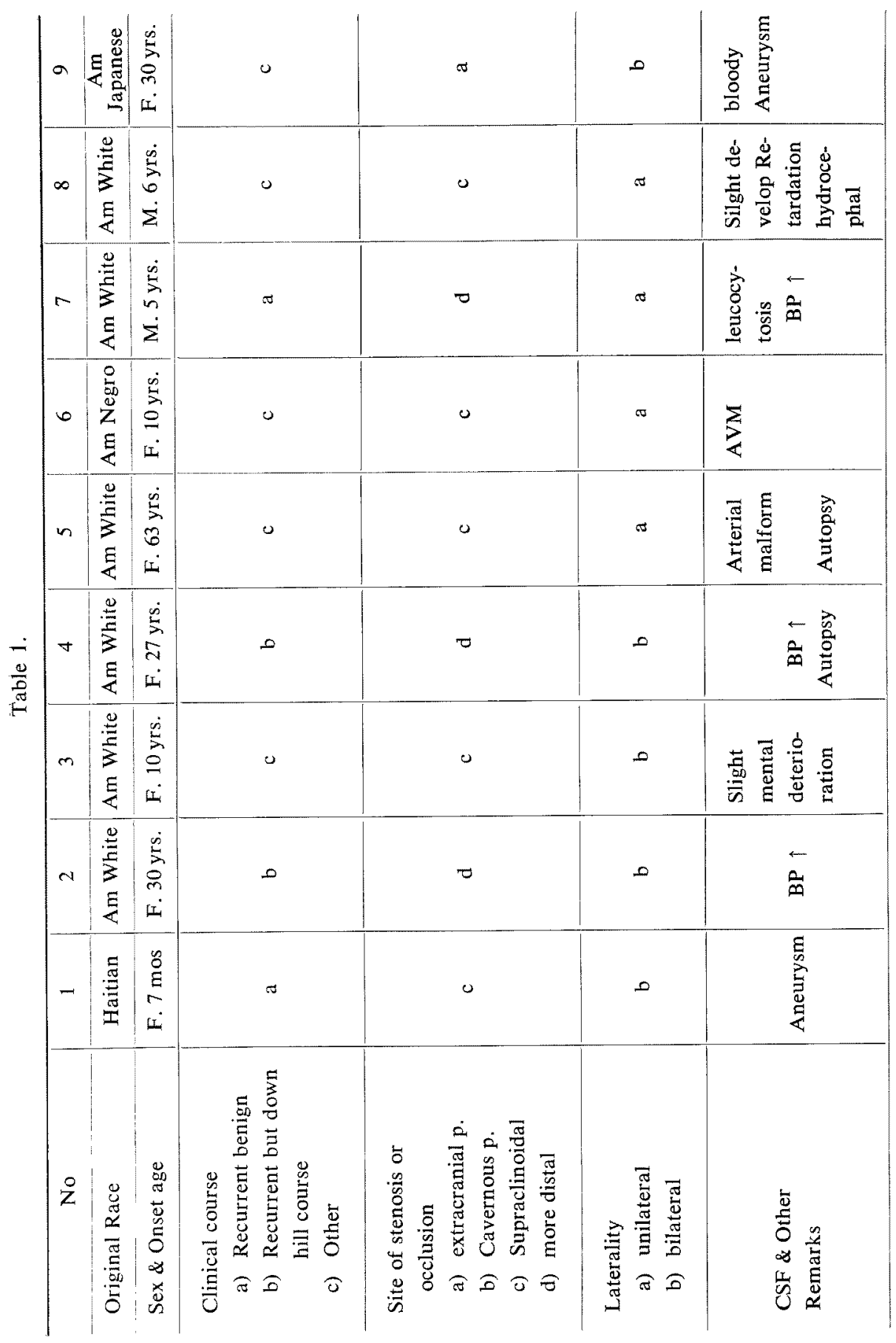




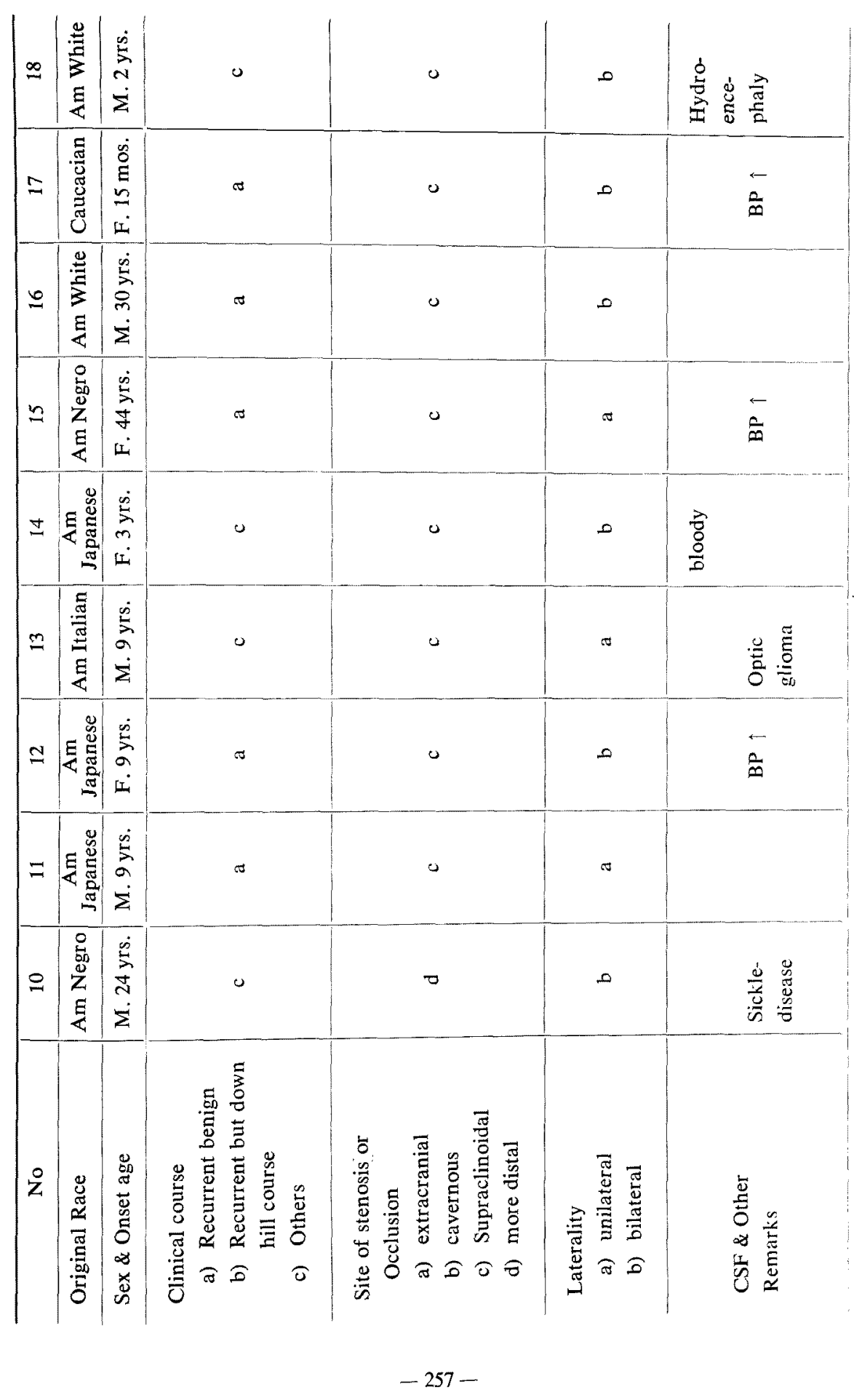


2) The angiograms and histories of 18 similar cases were obtained from 12 Institutions (Table 1).

Of these American cases which show similar angiographical findings each have narrowing or stenosis of the supraclinoid or more distal portion of whether the etiology is congenital or not.

Then we considered that these abnormal vascular networks in the cerebral arteries.

\title{
D-13. EEG Characteristics of the Occlusion of the Circle of Willis
}

\author{
Nobuo YosHII and Tatsuyuki Kudo \\ Div. of Neurological Surgery, Dept. of Surgery, School of Medicine, \\ Keio University
}

- Since the first introduction of the notion of "Insufficiency of the Circle of Willis" for the peculiar intracranial vascular nets which were thought to be caused by the occlusion or the stenosis of the Willis Circle and the main cerebral arterial trunks by Kudo in 1956, 12 cases have been extensively followed up in which 8 cases have been undergone the repeated EEG evaluation.

In 6 juvenile cases, all records are considered as abnormal, slow wave is observed in most cases during resting period and the prolonged effect of the marked slow waves is notified by the hyperventilation. One of the 2 adult cases, manifests the diffuse low voltage record with superimposed wave whereas the another shows normal tracing. No remarkable build-up is noted in both adult cases by hyperventilation.

\section{CASE PRESENTATION}

$8 \mathrm{y} / \mathrm{o}$ female who developed convulsive seizure at age 4 followed by rt. hemiparesis and motor aphasia manfests the central voltage emphasis and $\theta$ wave in resting recording of EEG and diffuse slow wave is beckoned for a few minutes by 2 minutes hyperventilation.

$14 \mathrm{y} / \mathrm{o}$ female who noted the onset of stepwisely progressive lt. hemiparesis at the age of 6 shows the diffuse high voltage slow wave of 3 minutes duration by hyperventilation and slow wave is shown in $\mathrm{rt}$. temporofrontal region in next 18 minutes.

$23 \mathrm{y} / \mathrm{o}$ female who manifested a sudden attack of disturbance of consciousness and lt. hemiplegia at age 13 shows diffuse episodic $\theta \sim \delta$ wave by hyperventilation.

By summarizing above-mentioned EEG recording, the appearance of the slow wave of considerable period after hyperventilation in cases of oclusion of 